\title{
Erbium-doped and Raman microlasers on a silicon chip fabricated by the sol-gel process
}

\author{
Lan Yang, ${ }^{\text {a) }}$ Tal Carmon, Bumki Min, Sean M. Spillane, and Kerry J. Vahala ${ }^{\text {b) }}$ \\ Department of Applied Physics, California Institute of Technology, Pasadena, California 91125
}

(Received 23 April 2004; accepted 26 January 2005; published online 25 February 2005)

\begin{abstract}
We report high-Q sol-gel microresonators on silicon chips, fabricated directly from a sol-gel layer deposited onto a silicon substrate. Quality factors as high as $2.5 \times 10^{7}$ at $1561 \mathrm{~nm}$ were obtained in toroidal microcavities formed of silica sol-gel, which allowed Raman lasing at absorbed pump powers below $1 \mathrm{~mW}$. Additionally, $\mathrm{Er}^{3+}$-doped microlasers were fabricated from $\mathrm{Er}^{3+}$-doped sol-gel layers with control of the laser dynamics possible by varying the erbium concentration of the starting sol-gel material. Continuous lasing with a threshold of $660 \mathrm{nW}$ for erbium-doped microlaser was also obtained. () 2005 American Institute of Physics. [DOI: 10.1063/1.1873043]
\end{abstract}

Recently, a chip-based silica resonator structure in the form of a microtoroid has demonstrated ultrahigh-Q-factors in the range of 100 million. ${ }^{1}$ By coating these high-Q microcavities with erbium-doped sol-gel films ${ }^{2}$ or by beginning with erbium implanted silica layers, ${ }^{3}$ low threshold rareearth-doped microlasers on-a-chip have been demonstrated. In another study, the realization of integrated Raman microlasers beginning with a layer of thermally-grown silica has been achieved. ${ }^{4}$ Microlasers on silicon chips are especially interesting because they are integrable with other optical or electric components. In this work, we demonstrate the possibility of making microlasers directly from the sol-gel layers on the silicon wafer. The sol-gel method for preparing silica and silicate from a metal alkoxide precursor provides a versatile and cost-effective way to fabricate various components on silicon chips. It combines control of composition and microstructure at the molecular level with the ability to shape materials in bulk, fiber, powder, and thin film forms. ${ }^{5}$ This technique allows a wide variety of thin films to be deposited on various substrates. In addition, optical devices, including buried-channel erbium-doped waveguide amplifiers, microlenses, one-dimensional photonic crystal devices and external-cavity distributed Bragg reflector (DBR) laser have been achieved using the sol-gel method. ${ }^{6-9}$ Here we report a new method to fabricate both erbium-doped microlasers and Raman microlasers on a silicon chip using sol-gel films as the base material for microtoroid formation. In one series of experiments, erbium-doped sol-gel films are used to create low threshold microlasers. In a second set of experiments, pure silica sol-gel layers are processed into ultrahigh-Q Raman microlasers. Both of these cases demonstrate the ability to use spin coating of sol-gel films as a processing alternative to deposition or oxidation methods for silica layer formation.

The sol-gel solution was prepared by hydrolyzing tetraethoxysilane (TEOS) using water with the molar ratio of water to TEOS around 1-2. Hydrochloric acid was added to make the solution in acid condition. $\mathrm{Er}^{3+}$ ions were introduced by adding $\operatorname{Er}(\mathrm{NO})_{3}$ to achieve the desired $\mathrm{Er}^{3+}$ concentration. After reaction at $70{ }^{\circ} \mathrm{C}$ for $3 \mathrm{~h}$, a viscous solution

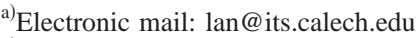

${ }^{\text {b)} U R L: \quad h t t p: / / w w w . i t s . c a l t e c h . e d u / \sim v a h a l a g r ; ~ e l e c t r o n i c ~ m a i l: ~}$ vahala@its.caltech.edu
}

was formed. The silica sol-gel film was then deposited on a silicon substrate by the spin-coating method. Different thicknesses of the sol-gel films were obtained by changing the viscosity of the sol-gel solution and spin speed, with multiple coating steps used to build up thicker layers. The thickness of the sol-gel film in this work was $1 \mu \mathrm{m}$ after three cycles of spin-coating. After each coating cycle, the chip was annealed at $1000{ }^{\circ} \mathrm{C}$ for $3 \mathrm{~h}$ to remove residual organics and solvents inside the sol-gel silica network. Following the spin-coating and anneal step, circular silica pads with diameter of 80 and 100 microns were created on the silicon wafer through a combination of optical lithography and buffered HF etching. Subsequently, these pads served as etch masks for isotropic silicon etching using $\mathrm{XeF}_{2}$. After this process, the silica disks are left supported by narrower silicon posts. As described in Ref. 1, a high power $\mathrm{CO}_{2}$ laser is then used to selectively reflow the silica sol-gel disks, during which surface tension causes the disks to collapse into toroids. The exceptionally smooth surface of the reflowed toroid cavities endows these structures with their high-Q properties. Figure 1 depicts the fabrication process flow of the microlasers beginning with the sol-gel layers on the Si wafer. Corresponding optical micrographs, taken after each processing step, are also shown in Fig. 1.

The principal diameters of the microtoroids studied in this work ranged from 50 to $60 \mu \mathrm{m}$. Tapered optical fibers were used to couple optical power both into and out of the resonator, with a fiber taper having a waist diameter of $1-2 \mu \mathrm{m}$ used in order to couple to the resonator with high efficiency. ${ }^{10}$ To vary the air gap between the microcavity and the taper, the sample chips were mounted on a three-axis translator for position control. A tunable, single-frequency, narrow linewidth $(<300 \mathrm{kHz})$ external-cavity laser emitting in the $1480 \mathrm{~nm}$ band was used to pump the erbium-doped microlaser. The fiber output was connected to a 90/10 fused fiber coupler, with the $10 \%$ port connected to an optical spectrum analyzer to monitor both the pump and signal spectra. The $90 \%$ port was connected to an oscilloscope to observe the laser dynamics. The pump laser was scanned repeatedly around a single whispering gallery mode. The absorbed pump power was measured as the difference of launched power into the taper and the transmitted power after the taper. Figure 2 shows a laser emission spectrum for $\mathrm{CW}$ (continuous wave) operation of an $\mathrm{Er}^{3+}$-doped microtoroid 


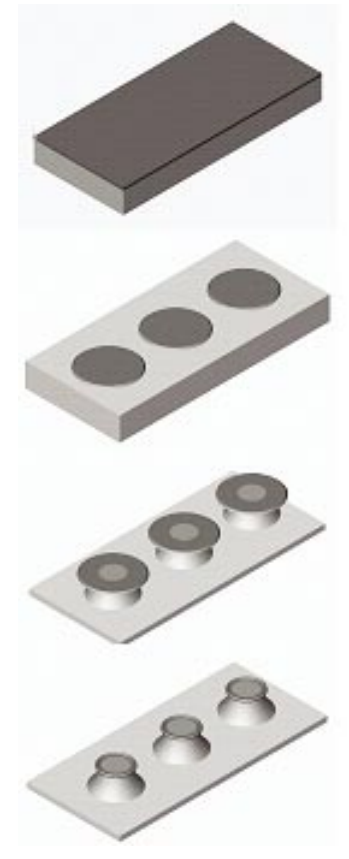

(a)

(b)

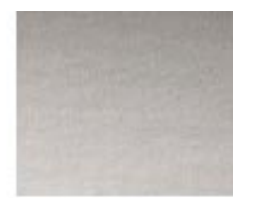

(c)

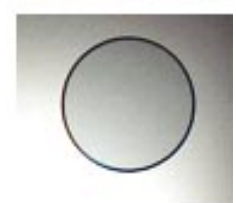

(d)

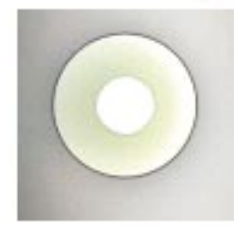

FIG. 1. (Color) Schematic process flow for creation of solgel microcavities on a $\mathrm{Si}$ wafer (left) and photomicrograph plan view (right) after each step. (a) Sol-gel layer is spun on Si wafer; (b) circular pads are etched; (c) $\mathrm{XeF}_{2}$ isotropic silicon etch; (d) $\mathrm{CO}_{2}$ laser reflow.

laser (optical spectrum analyzer resolution of $0.5 \mathrm{~nm}$ ) with a micrograph of the fiber-coupled microlaser presented in the inset. Single-mode operation could be obtained by proper choice of the coupling condition. The green ring around the toroid periphery is due to upconverted photoluminescence from $\mathrm{Er}^{3+}$.

A theoretical analysis shows that the threshold power is minimized at a certain erbium ion concentration. ${ }^{11}$ This optimal erbium ion concentration depends, in turn, on the intrinsic quality factor of the pump mode. ${ }^{11}$ In the low concentration limit, the threshold power increases sharply because $\mathrm{Er}^{3+}$ ions are not able to give sufficient gain required for loss compensation; while in high concentration limit, the threshold again increases due to increases of concentrationdependent loss mechanism, such as upconversion and ionpair induced quenching. For further details on this theoretical optimum threshold, we refer the reader to Ref. 11. We found

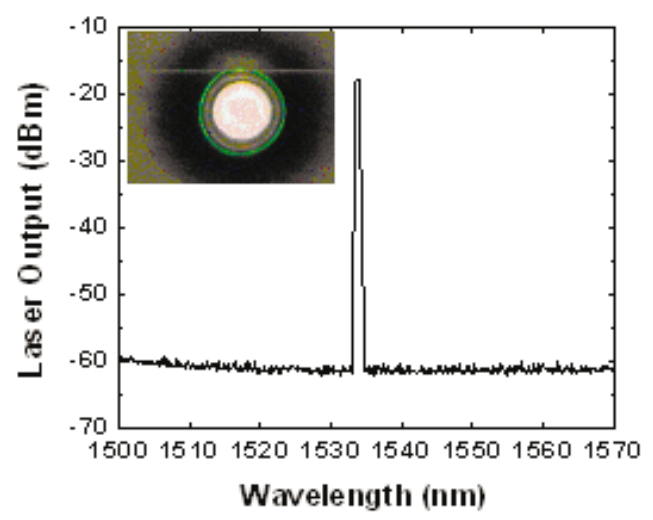

FIG. 2. (Color) Typical laser spectrum of an $\mathrm{Er}^{3+}$-doped solgel silica microtoroid laser. The inset is a photomicrograph top view of an $\mathrm{Er}^{3+}$-doped solgel silica microtoroid laser with principal diameter of $60 \mu \mathrm{m}$ coupled by a fiber taper. The green ring is due to upconverted photoluminescence from

$\mathrm{Er}^{3+}$.
Downloaded 14 Dec 2005 to 131.215 .225 .171 . Redistribution subject to AlP license or copyright, see http://apl.aip.org/apl/copyright.jsp

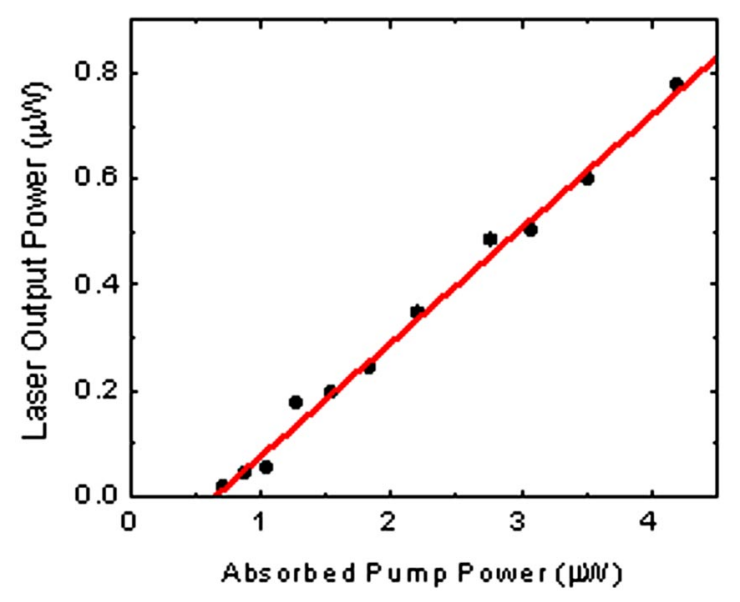

FIG. 3. (Color online) Measured laser output power plotted vs the absorbed pump power for a microtoroid laser with principal diameter of $60 \mu \mathrm{m}$. The lasing threshold is $660 \mathrm{nW}$ with pump wavelength at $1442 \mathrm{~nm}$ and lasing wavelength at $1553 \mathrm{~nm}$.

that for a toroid with a principal diameter of $60 \mu \mathrm{m}$ and intrinsic quality factor (pump mode) between $5 \times 10^{6}$ and 1 $\times 10^{7}$, thresholds in the range of $400-600 \mathrm{nW}$ can be achieved with an $\mathrm{Er}^{3+}$ concentration of $2 \times 10^{19} \mathrm{~cm}^{-3}$. Figure 3 shows the measured laser output power plotted versus the absorbed pump power from a microtorid with properties as described above. The threshold was estimated to be as low as $660 \mathrm{nW}$, which is about three times lower than that of the most recently reported Er-implanted microtoroid resonators. ${ }^{3}$ This ultralow threshold originates from the small mode volume, high quality factor of the microcavity, and homogeneous distribution of the $\mathrm{Er}^{3+}$ inside the cavity, which enable the optimized overlap between the active region and the pump modes. Above threshold, the laser output power increases linearly with the absorbed pump power, as expected.

By varying the erbium concentration in the starting solgel solution, we could adjust the final doping concentration in the microcavities, which ultimately modifies the laser dynamics as previously described for the case of microsphere lasers. $^{12}$ In particular, for a heavily doped $\left(\mathrm{Er}_{2} \mathrm{O}_{3}\right.$ $\sim 0.15 \mathrm{~mol} \%$ ) microcavity, pulsation behavior, as opposed to $\mathrm{CW}$ operation, is observed. The pulse repetition rate is $0.9 \mathrm{MHz}$ at a laser output power of $3.8 \mu \mathrm{W}$ and as described in Ref. 12 is attributed to the presence of saturable absorption in the cavity owing to unpumped or incompletelypumped erbium regions and ion-pair induced quenching.

Raman oscillation at low pump powers using ultrahigh-Q microcavities is possible even in extremely linear media such as silica. ${ }^{13}$ To investigate the possibility of Raman oscillation in sol-gel microcavities, microtoroids were fabricated from an undoped sol-gel layer. The quality factor of the initial microdisk preform was $7.1 \times 10^{4}$, whereas after the $\mathrm{CO}_{2}$ laser reflow the microtoroid achieved quality factors as high as $2.5 \times 10^{7}$ at $1561 \mathrm{~nm}$. This corresponds to a waveguide loss of $0.009 \mathrm{~dB} / \mathrm{cm}$, which is the lowest loss reported to date for sol-gel silica-on-silicon technology. ${ }^{14,15}$ These high quality factors allow the observation of Raman emission with threshold pump powers below a milliwatt. In this case, a single-frequency tunable externalcavity laser operating around $1550 \mathrm{~nm}$ band was used to pump the microcavity. Figure 4 shows a typical Raman laser spectrum taken on a microtoroid with a quality factor of $2.5 \times 10^{7}$ at $1561 \mathrm{~nm}$. In the figure both pump wave at 


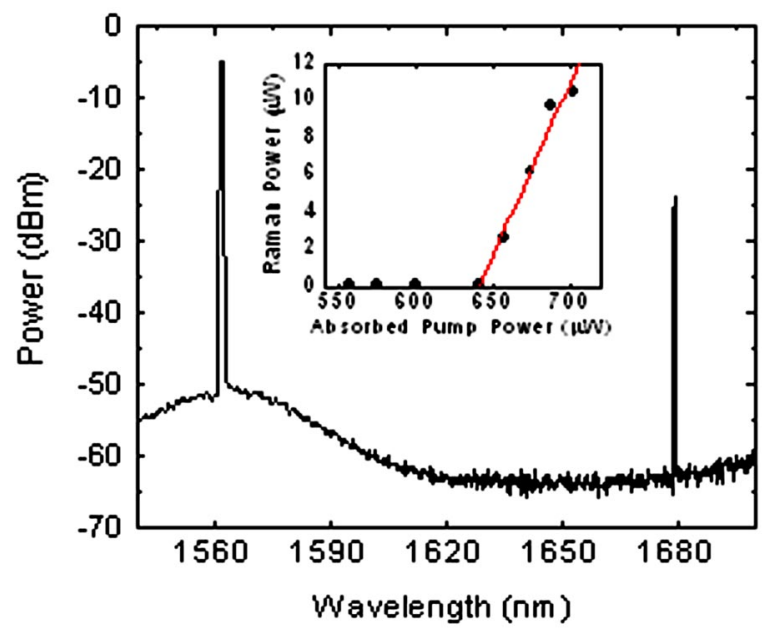

FIG. 4. (Color online) Raman emission spectrum of an undoped microtoroid with principal diameter of $49 \mu \mathrm{m}$. The pump wavelength is at $1561 \mathrm{~nm}$ and Raman oscillation occurs at $1679 \mathrm{~nm}$. The inset shows the measured Raman laser output power vs the absorbed pump power.

$1561 \mathrm{~nm}$ and Raman lasing at $1679 \mathrm{~nm}$ are visible. The inset in Fig. 4 presents a measurement of Raman lasing power as a function of absorbed pump power, which shows a submilliwatt threshold of $640 \mu \mathrm{W}$.

In conclusion, we have demonstrated that by using the sol-gel process both $\mathrm{Er}^{3+}$-doped microlasers and Raman microlasers can be fabricated on a silicon chip. Small mode volume and very low loss of the modes made record threshold in the sub- $\mu \mathrm{W}$ range possible for the $\mathrm{Er}^{3+}$-doped lasers tested. By varying the doping concentration of erbium in the sol-gel layers, the laser dynamics can also be controlled. Additionally, the $\mathrm{CO}_{2}$ densification and reflow process dramatically lowers the optical loss of the sol-gel whispering gallery waveguide, resulting in quality factors as high as
$2.5 \times 10^{7}$ at $1561 \mathrm{~nm}$ for an undoped cavity. The sol-gel process provides a versatile way to create silica-based devices with precise control of the composition. The results demonstrate the great potential of sol-gel technology in fabrication of high optical quality materials for integrated optical components on silicon chips.

This work was supported by the Defense Advanced Research Project Agency, the National Science Foundation, and the Caltech Lee Center.

${ }^{1}$ D. K. Armani, T. J. Kippenberg, S. M. Spillane, and K. J. Vahala, Nature (London) 421, 925 (2003).

${ }^{2}$ L. Yang, D. K. Armani, and K. J. Vahala, Appl. Phys. Lett. 83, 825 (2003).

${ }^{3}$ A. Polman, B. Min, J. Kalkman, T. J. Kippenberg, and K. J. Vahala, Appl. Phys. Lett. 7, 1037 (2004).

${ }^{4}$ T. J. Kippenberg, S. M. Spillane, D. K. Armani, and K. J. Vahala, Opt. Lett. 29, 1224 (2004).

${ }^{5}$ Rustum Roy, Science 238, 1664 (1987).

${ }^{6}$ W. Huang and R. R. A. Syms, J. Lightwave Technol. 21, 1339 (2003).

${ }^{7}$ M. He, X. C. Yuan, N. Q. Ngo, J. Bu, and V. Kudryashow, Opt. Lett. 28, 731 (2003).

${ }^{8}$ Kevin M. Chen, Andew W. Sparks, Hsin-Chiao Luan, Desmond R. Lim, Kazumi Wade, and Lionel C. Kimerling, Appl. Phys. Lett. 75, 3805 (1999).

${ }^{9}$ M. A. Fardad, H. Luo, Y. Beregovski, and M. Fallahi, Opt. Lett. 24, 460 (1999).

${ }^{10}$ S. M. Spillane, T. J. Kippenberg, O. J. Painter, and K. J. Vahala, Phys. Rev. Lett. 91, 043902 (2003).

${ }^{11}$ B. Min, T. J. Kippenberg, L. Yang, K. J. Vahala, J. Kalkman, and A. Polman, Phys. Rev. A 70, 033803 (2004).

${ }^{12}$ L. Yang and K. J. Vahala, Opt. Lett. 28, 592 (2003).

${ }^{13}$ S. M. Spillane, T. J. Kippenberg, and K. J. Vahala, Nature (London) $4 \mathbf{4 1 5}$, 621 (2002).

${ }^{14}$ R. A. Syms, V. Schneider, W. Huang, and A. S. Holms, Electron. Lett. 31, 1833 (1995).

${ }^{15}$ J. Bellessa, S. Rabaste, J. C. Plenet, J. Dumas, J. Mugnier, and O. Marty, Appl. Phys. Lett. 79, 14 (2001). 